\title{
Maladaptive role of neutrophil extracellular traps in pathogen-induced lung injury
}

\author{
Emma Lefrançais, ${ }^{1}$ Beñat Mallavia, ${ }^{1}$ Hanjing Zhuo, ${ }^{1}$ Carolyn S. Calfee, ${ }^{1}$ and Mark R. Looney,2 \\ 'Department of Medicine and 'Department of Laboratory Medicine, UCSF, San Francisco, California, USA.
}

\begin{abstract}
Neutrophils dominate the early immune response in pathogen-induced acute lung injury, but efforts to harness their responses have not led to therapeutic advancements. Neutrophil extracellular traps (NETs) have been proposed as an innate defense mechanism responsible for pathogen clearance, but there are concerns that NETs may induce collateral damage to host tissues. Here, we detected NETs in abundance in mouse models of severe bacterial pneumonia/acute lung injury and in human subjects with acute respiratory distress syndrome (ARDS) from pneumonia or sepsis. Decreasing NETs reduced lung injury and improved survival after DNase I treatment or with partial protein arginine deiminase 4 deficiency $\left(P A D 4^{+/-}\right)$. Complete PAD4 deficiency $\left(P A D 4^{-/-}\right)$reduced NETs and lung injury but was counterbalanced by increased bacterial load and inflammation. Importantly, we discovered that the lipoxin pathway could be a potent modulator of NET formation, and that mice deficient in the lipoxin receptor $\left(\mathrm{Fpr}^{-\mathrm{I}^{-}}\right)$produced excess NETs leading to increased lung injury and mortality. Lastly, we observed in humans that increased plasma NETs were associated with ARDS severity and mortality, and lower plasma DNase I levels were associated with the development of sepsis-induced ARDS. We conclude that a critical balance of NETs is necessary to prevent lung injury and to maintain microbial control, which has important therapeutic implications.
\end{abstract}

Conflict of interest: The authors have declared that no conflict of interest exists.

Submitted: October 20, 2017 Accepted: December 28, 2017 Published: February 8, 2018

\section{Reference information:} JCI Insight. 2018;3(3):e98178. https:// doi.org/10.1172/jii.insight.98178.

\section{Introduction}

The lung is continuously exposed to the external environment and therefore must be armed to identify and eliminate pathogens. Acute respiratory infections remain a burden worldwide, affecting millions of people annually and resulting in approximately 4 million deaths (1). Although an effective inflammatory response orchestrated by resident and recruited cells in the lung is required to protect against invading pathogens, a prolonged or exaggerated response can damage lung tissue. Thus, the immune response represents a balancing act between the elimination of the infection and immune-mediated pulmonary injury.

Clinically, immune-mediated lung injury after acute sterile or microbial insults manifests as the acute respiratory distress syndrome (ARDS), a disorder initially described 50 years ago (2). Pneumonia and sepsis are the leading causes of this severe lung disorder that is associated with a high mortality rate approaching $40 \%$ (3). Despite refinements in the delivery of supportive care, including the use of protective mechanical ventilation and maintaining an even fluid balance, it is difficult to effectively prevent or treat ARDS. Animal models of a variety of causes of ARDS have consistently identified the neutrophil as a central cellular mediator of the early, innate immune response, and neutrophils abundantly accumulate in the lung capillaries and alveolar spaces of patients with ARDS (4).

More recently, neutrophils were described as releasing their nuclear contents into the extracellular spaces in response to a variety of challenges in a process termed neutrophil extracellular trap (NET) formation (5). NETs are filamentous chromatin fibers decorated with neutrophil-derived antimicrobial proteins generated by a process that is dependent on ROS and deimination of critical amino acids on histones, and may or may not require neutrophil death (6). NETs serve as an emergency first-line defense mechanism to trap and kill extracellular bacteria and other pathogens during serious infections; however, NETs are increasingly implicated in a variety of noninfectious diseases (7). NETs have been associated with inflammation and tissue injury $(8,9)$ but are also potentially protective against infection (10). Adding to the ambiguity concerning NETs is the elucidation of mechanisms to regulate NETs through DNase production by the host $(11,12)$ or pathogens $(13)$ to 
protect against excessive damage or to evade entrapment, respectively. Particularly during infection-associated ARDS, it is unknown if NETs are required to help contain and clear infections or perhaps contribute to the lung barrier disruption that is the hallmark of ARDS. Indeed, NETs can immobilize or trap various pathogens, subsequently counteracting the dissemination of infections and ultimately promoting pathogen killing $(13,14)$. However, the excessive production of NETs, and particularly NET-bound components, may produce harmful effects $(15,16)$. In sterile models of lung injury, NETs promote alveolar-capillary injury, since targeting NETs decreases the severity of lung injury and protects from mortality (17-19).

Here, we investigated the in vivo significance of NETs in mouse models of acute lung injury produced from 2 common respiratory pathogens, methicillin-resistant Staphylococcus aureus (MRSA) and Pseudomonas aeruginosa. MRSA and $P$. aeruginosa are frequent causes of nosocomial and ventilator-associated pneumonia (20), and MRSA is increasingly implicated in outbreaks of community-acquired infections (21). We tested mechanisms to prevent NET formation (protein arginine deiminase 4 [PAD4] inhibition), potentially novel mechanisms to regulate NETs (lipoxins), and the dismantling of existing NETs (DNase I) for their impact on bacterial clearance and lung barrier disruption. Lastly, we tested the in vivo significance of NETs and their relationship to DNase I levels using unique human biological samples from critically ill patients with pneumonia- and sepsis-induced ARDS.

\section{Results}

NETs are formed during pneumonia-induced lung injury. We developed a mouse model of pneumonia-induced acute lung injury (ALI) in which a clinical strain of MRSA (USA300) (22) is instilled intratracheally (i.t.). We tested different inocula of MRSA, and settled on a dose of $5 \times 10^{7} \mathrm{CFU} /$ mouse, which produces severe ALI with increased lung permeability, assessed by bronchoalveolar lavage (BAL) total protein levels (Figure 1A), lung vascular permeability to albumin (Figure 1B), and increased extravascular lung water (Figure 1C). Analysis of BAL cells also reveals striking neutrophilia ( $>90 \%$ of BAL cells) at 10 to 24 hours after infection (Figure 1, D-F). We used our custom neutrophil elastase-DNA (NE-DNA) ELISA to measure soluble NETs in the BAL (Figure $1 \mathrm{G}$ ) and plasma (Figure $1 \mathrm{H}$ ) and found that NETs are first formed in the alveolar spaces starting at approximately $6-8$ hours after infection and continue to increase, paralleling the increase in lung permeability (Figure 1I). We also tested infection with $P$. aeruginosa (PAO1) and found a dose-dependent increase in inflammatory cell recruitment to the lung (Figure 1J), lung permeability (Figure $1 \mathrm{~K}$ ), and abundant NET production in BAL (Figure 1L) and plasma (Figure 1M).

Since the BAL procedure can disrupt NET structures, and since NETs can be difficult to detect in lung sections, we developed a potentially novel methodology to detect NET structures by immunofluorescence. We discovered that neutrophils recovered from the BAL of infected mice produce NETs ex vivo under cell culture conditions. We detected NETs in the cultured BAL of MRSA- and PAO1-challenged mice by colocalizing extracellular DNA, neutrophil elastase, and extracellular histones (Figure 2, A and B), similar to BAL obtained from mice challenged with MIP-2 (i.t.) to recruit neutrophils and subsequently stimulated with PMA (Supplemental Figure 1A). NETs were not observed in unstimulated neutrophil-rich BAL (Supplemental Figure 1B) or in BAL neutrophils undergoing necrosis induced by freeze and thaw cycles (Supplemental Figure 1C).

Dynamic formation of in vivo NETs during pneumonia. To explore the production of NETs in their physiological environment during pneumonia, we used lung 2-photon intravital microscopy to determine the spatial and temporal formation of NETs within the pulmonary microcirculation. To track neutrophils, we used LysM-eGFP mice (23) or $m T m G$ or $n T n G$ mice (24) crossed with MRP8-Cre mice (25). In the latter, membrane or nuclear $\mathrm{GFP}^{+}$neutrophils can be precisely tracked on the background of tomato ${ }^{+}$stromal cells. Infection with MRSA rapidly induced neutrophil recruitment and sequestration in the lung beginning at 2 hours after MRSA infection (Figure 2, C and D and Supplemental Video 1), consistent with the BAL fluid analysis (Figure 1, D-F). Neutrophils were observed to internalize SYTO Red-stained MRSA at early time points after infection (Supplemental Figure 1D). With PAO1 challenge, we observed neutrophil swarming, and the use of an mCherry fluorescent strain revealed that clusters of neutrophils were formed around the bacteria (Figure $2 \mathrm{E}$ and Supplemental Video 1).

Using the cell-impermeant DNA dye SYTOX Green in MRP8-mTmG or $n T n G$ mice, we examined cell death and DNA release in the lung. Dynamic NET formation, characterized by SYTOX Green-positive strands, was observed being released from $\mathrm{GFP}^{+}$neutrophils at 3 hours after PAO1 infection (Figure $2 \mathrm{~F}$ ), but were not visible in noninfected animals (data not shown). The extracellular DNA protrusions were detected both in the lung vasculature (Figure 2F and Supplemental Video 2) and in the alveolar spaces where neutro- 
A

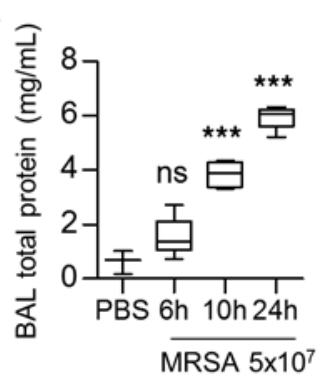

B

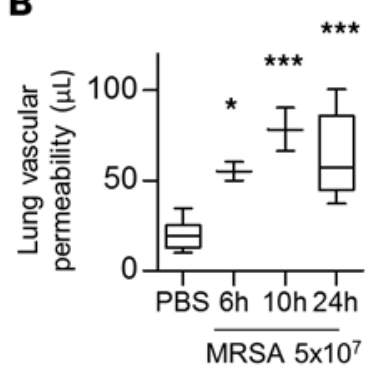

C

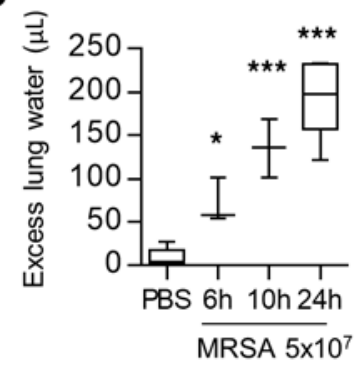

D

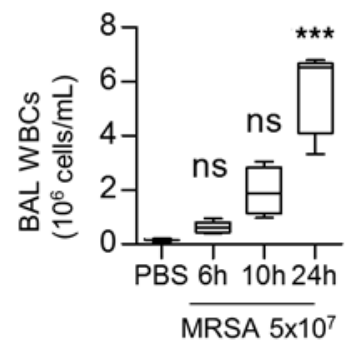

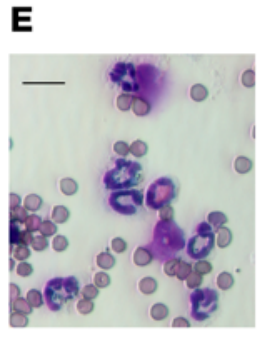

$\mathbf{F}$

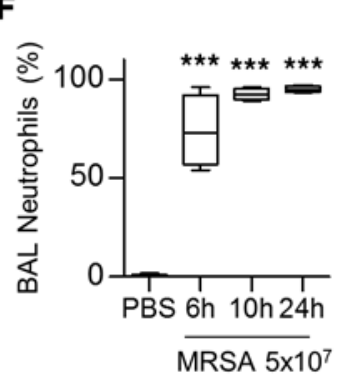

G

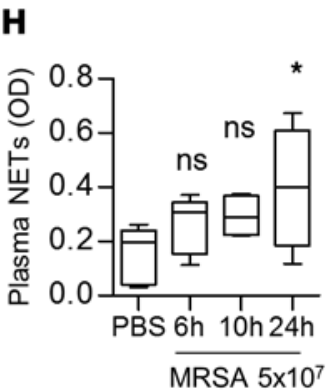

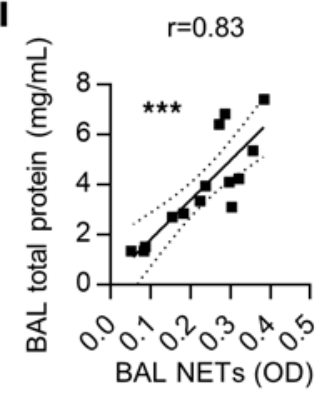
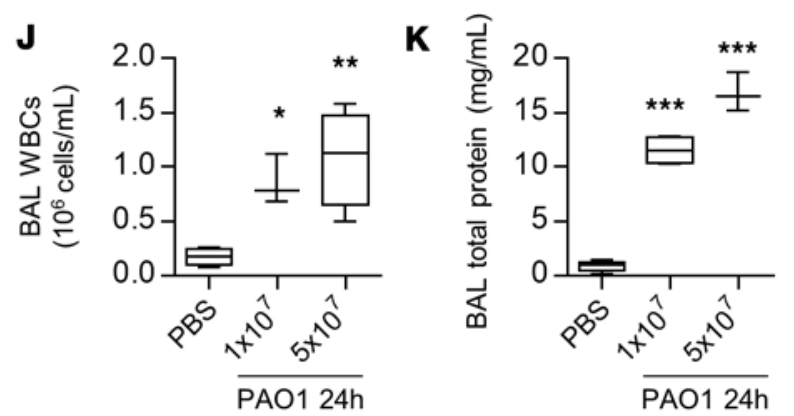

$\mathbf{L}$

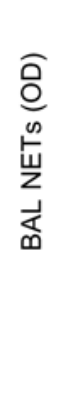

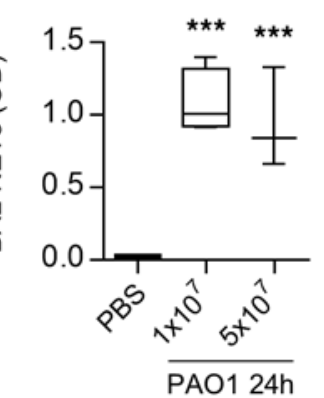

M

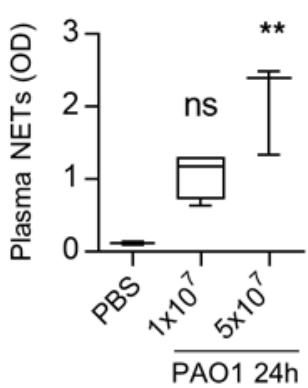

Figure 1. Neutrophil extracellular traps (NETs) are elaborated during MRSA- and PA01-induced lung injury models. (A) Mice were instilled intratracheally (i.t.) with methicillin-resistant Staphylococcus aureus (MRSA) at $5 \times 10^{7} \mathrm{CFU} /$ mouse and sacrificed at indicated time points $(n=3-12)$. (A) Bronchoalveolar lavage (BAL) total protein concentration, (B) lung vascular permeability to radioactive albumin, (C) excess lung water, and (D) BAL WBC were all increased after infection. (E) BAL cytospin and (F) differential counts are composed mainly of neutrophils. Scale bar: $20 \mu \mathrm{m}$. (C) NETs (neutrophil elastase-DNA complexes) in BAL and (H) plasma. (I) BAL NETs and protein concentration are positively correlated ( $r$ = Pearson's correlation coefficient). (J-M) Mice were instilled i.t. with Pseudomonas aeruginosa strain PA01 at $1 \times 10^{7}$ or $5 \times 10^{7} \mathrm{CFU} /$ mouse and sacrificed at 24 hours $(n=3-4)$. (J) BAL WBC, (K) BAL total protein concentration, (L) BAL NETs, and (M) plasma NETs were all increased after infection. Data were analyzed using 1-way ANOVA and Dunnett's post test. ${ }^{*} P \leq 0.05,{ }^{* *} P \leq 0.01,{ }^{* *} P \leq 0.001$. ns, not significant.

phils were recruited (Figure 2G, Supplemental Figure 1E, and Supplemental Video 3). Using mCherry PAO1, we found that the bacteria colocalized with extracellular DNA strands in the lung (Figure 2H). Overall, the intravital microscopy data demonstrate that lung infection with bacteria rapidly induces the recruitment and swarming of neutrophils and the release of NETs in the lung vasculature and the alveolar spaces. Of note, NETs detected in the alveolar space were stable for hours of imaging, while NETs in the circulation spontaneously cleared in minutes (Supplemental Video 2). These observations are perhaps explained by the presence of blood flow, but another hypothesis is that intravascular NETs are rapidly degraded by circulating DNases.

Mice with impaired NETosis have reduced lung injury but impaired bacterial clearance. Based on results demonstrating robust NET formation in the lung, we next evaluated the in vivo contribution of NETs to tissue injury and bacterial clearance. We infected $P A D 4^{-1-}$ mice in which histone citrullination and chromatin decondensation, believed to be required for NET formation, is impaired (26, 27). We confirmed that NET formation was reduced in $P A D 4^{-/-}$bone marrow neutrophils (Figure 3, A-C). NETs in BAL (Figure 3, D-F) and plasma (Supplemental Figure 2A) from $P A D 4^{-1-}$ mice were reduced, but not completely abolished, even though citrullination was eliminated (Figure 3, E and G and Supplemental Figure 2B). These data (ELISA and immunofluorescence) suggest that in vivo NET formation is partially independent of citrullination. 
BAL, MRSA challenge $\left(5 \times 10^{7} \mathrm{cfu}\right.$, i.t. $\left.-15 \mathrm{~h}\right)$
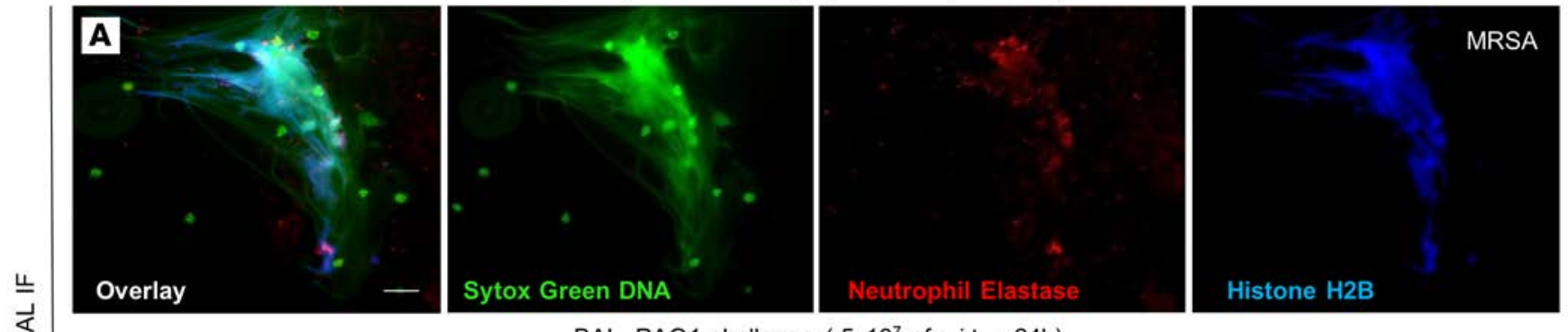

BAL, PAO1 challenge $\left(5 \times 10^{7}\right.$ cfu, i.t. $\left.-24 \mathrm{~h}\right)$
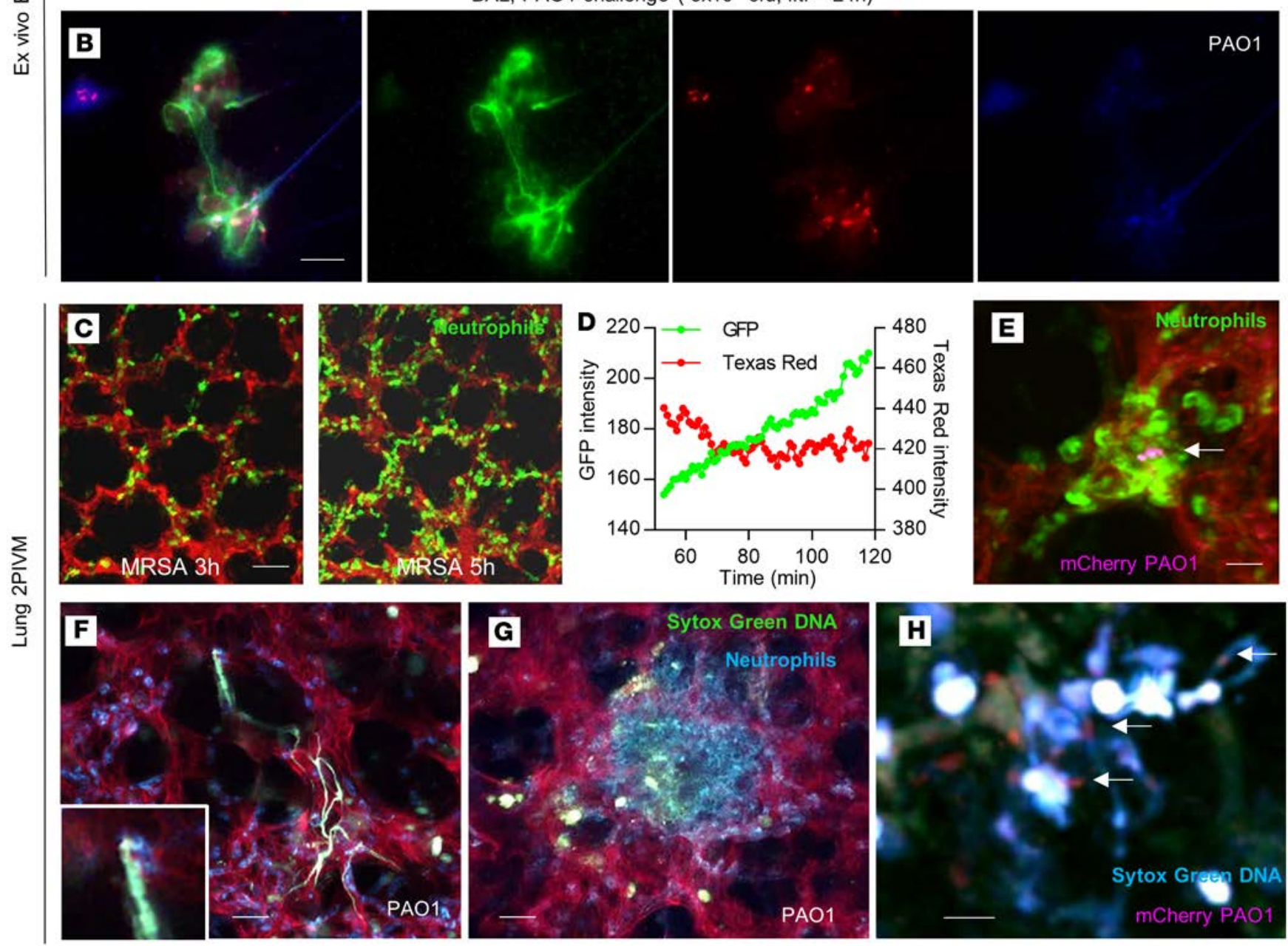

Figure 2. NETs are visualized ex vivo and in vivo in infected lungs. (A and B) Bronchoalveolar lavage (BAL) from mice infected with (A) methicillin-resistant Staphylococcus aureus (MRSA) or (B) Pseudomonas aeruginosa strain PA01 was settled on a slide and stained ex vivo with SYTOX Green DNA dye (green), neutrophil elastase (NE) antibody (red), and histone H2B antibody (blue). (C-H) Lung 2-photon intravital microscopy. (C) LysM-GFP mice (green neutrophils) were challenged with MRSA ( $2 \times 10^{7} \mathrm{CFU}$, i.t.), injected with Texas Red-dextran i.v. to stain the vasculature, and observed from 3 to 5 hours after infection. (D) GFP and Texas Red signals were quantified, showing neutrophils accumulating in the lungs. (E) MRP8-mTmG mice (red vasculature, green neutrophils) were challenged i.t. with $5 \times 10^{6} \mathrm{CFU}$ mCherry-PA01 (pink, arrows) and observed from 3 to 5 hours after the infection. (F and G) MRP8-mTmG mice (red vasculature, blue neutrophils) were challenged with PAO1 ( $5 \times 10^{6} \mathrm{CFU}$, i.t.) and observed from 3 to 5 hours after the infection. Extracellular DNA was stained with SYTOX Green. (H) WT mice injected with FITC-dextran (green vasculature) were challenged i.t. with $5 \times 10^{6} \mathrm{CFU}$ mCherry-PA01 (pink, arrows) and observed 17 hours after the infection. Extracellular DNA was stained with SYTOX Blue. Scale bars: $20 \mu \mathrm{m}$ (A and $\mathbf{H}), 10 \mu \mathrm{m}$ (B and E), and $50 \mu \mathrm{m}(\mathbf{C}, \mathbf{F}$, and $\mathbf{G})$.

NET reduction in $P A D 4^{-/-}$mice reduced lung injury (Figure $3 \mathrm{H}$ ); however, $P A D 4^{-/-}$mice had higher bacterial counts in the lung (Figure 3I) and higher levels of inflammatory cytokines (IL-1 $\beta$ and IL-6) compared with WT mice (Figure 3, J and K), which supports the role of NETs in bacterial clearance. Because of this complex balance, the overall survival rate was unchanged in the $P A D 4^{-/-}$mice (Figure $3 \mathrm{~L}$ ). However, $P A D 4^{+/-}$mice (littermates) had a surprisingly improved survival rate after MRSA challenge. These mice have 
an intermediate phenotype between WT and knockout, with intermediate NET production (Figure 3, F and G and Supplemental Figure 2, A and B), intermediate lung permeability (Figure 3H), lung bacterial load (Figure 3I), and inflammatory cytokine production (Figure 3, J and K). Reduction of PAD4 in heterozygous and knockout mice did not change the number of total leukocytes or neutrophils recruited to the lung (Supplemental Figure 2, C and D), but macrophages were decreased (Supplemental Figure 2E) and there was a slight increase in the cytokines IL-12 and IFN- $\gamma$ (Supplemental Figure 2, F and G). These changes were more pronounced in heterozygous mice, and a modification of immune cell profile and the inflammatory environment may explain the advantage of the heterozygous mice over WT and knockout mice.

Pharmacologic PAD inhibition reduces histone citrullination and inflammatory cytokines, but does not affect lung injury. We also tested a pharmacologic approach to inhibit citrullination and NETosis by treating mice with Cl-amidine (i.p.), a nonselective PAD inhibitor, 3 hours after infection (Supplemental Figure 3A). Citrullinated histone-DNA conjugates were decreased in the BAL fluid (Supplemental Figure 3B), but Cl-amidine treatment failed to decrease NE-DNA complexes in the lung (Supplemental Figure 3C), had no effect on lung injury or inflammatory cell recruitment to the lung, and no effect on bacterial clearance (Supplemental Figure 3, E-G). Plasma NETs decreased after Cl-amidine treatment (Supplemental Figure 3D), which may have led to less temperature loss (Supplemental Figure $3 \mathrm{H}$ ) and lower levels of many inflammatory cytokines (Supplemental Figure 3, I-O).

NET degradation with DNase I reduces lung injury and improves survival. In genetically modified mouse models like $P A D 4^{-1-}$, the reduction of NETs from the onset of the infection may compromise the ability to contain bacteria. We therefore reasoned that disrupting NETs after their production could limit the extent of lung injury, and that in conjunction with antibiotics to control lung infection, anti-NET therapy might effectively reduce the progression of pneumonia to acute lung injury. To test this hypothesis, we treated MRSA-infected mice with DNase I or diluent control administered by i.t. instillation 2 hours after infection. We tested for the presence of NETs and lung injury 8 hours after the onset of infection (Figure 4A). Since the exogenously administered DNase I would confound BAL fluid total protein measurements, we instead measured BAL albumin as an indicator of lung vascular permeability. DNase I treatment effectively reduced NE-DNA complexes in the BAL (Figure 4B) without affecting cell recruitment to the lung (Figure 4C). DNase I treatment also reduced BAL albumin leakage (Figures 4D), vascular permeability to radioactive albumin (Figure 4E), and extravascular lung water (Figure 4F). In contrast to $P A D 4^{-1-}$ mice (Figure 3I), degradation of NETs by DNase I treatment did not increase the bacterial counts in the lung or blood (Figure 4, G and H). To test the effect of DNase I on mortality, we treated mice every $8-10$ hours to maintain efficient NET degradation. DNase I treatment improved mortality with or without the addition of a pathogen-specific antibiotic (vancomycin) (Figure 4, I-L). In combination with vancomycin, DNase I treatment also improved the physiologic condition of the animals, as shown by reduced hypothermia (Figure 4M) and reduced blood lactate levels (Figure 4N).

The lipoxin pathway regulates NET production. We have previously demonstrated that the lipoxin pathway, and specifically formyl peptide receptor 2 (Fpr2) agonists including the antiinflammatory, proresolving mediator 15-epi-lipoxin $\mathrm{A}_{4}$, effectively reduces lung inflammation and injury after i.t. LPS challenge and in a mouse model of transfusion-related ALI (TRALI) (23). We hypothesize that lipoxin $\mathrm{A}_{4}$ and other mediators in this class that signal through Fpr2 can be potent modulators of NET formation. To test this hypothesis, we first tested neutrophils incubated with Fpr2 agonists before stimulation with PMA or MRSA. Lipoxin $\mathrm{A}_{4}$ reduced the PMA- and MRSA-induced formation of NETs in WT but not in Fpr2-/- neutrophils, which establishes that this pathway is a feasible target (Figure 5, A and B). Since phenol-soluble modulin (PSM) toxins from MRSA are potent Fpr2 agonists (28), we tested if MRSA could have an effect on NETosis via Fpr2. However, $\mathrm{Fpr}^{-/-}$neutrophils produced the same amount of NETs as WT neutrophils after MRSA stimulation (Figure 5B). In vivo, we compared development of lung injury and production of NETs in $\mathrm{Fpr}^{2^{--}}$mice. We observed a striking phenotype for increased NET production, lung injury, and mortality in mice deficient in Fpr2 (Figure 5, $\mathrm{C}-\mathrm{F}$ and $\mathrm{H}$ ), which is consistent with our hypothesis that Fpr2 signaling provides an endogenous brake signal on the production of NETs. It is important to note that Fpr2 deficiency increased leukocyte infiltration into the lung (Figure 5F), which may partly explain the increase in BAL NETs (Figure 5D) observed in vivo. Bacterial burden in the lungs was unaffected by Fpr2 deletion (Figure 5G).

NETs are present in ARDS and correlate with ARDS severity and mortality. To establish the clinical significance of our findings, we measured NETs in human samples from critically ill patients, many of whom have ARDS or are at risk for ARDS from the Early Assessment of Renal and Lung Injury (EARLI) study at UCSF. The EARLI study enrolls patients in the emergency room at the time of triage to the intensive 
A

$\mathrm{PAD}^{+/+}$or PAD4 ${ }^{-/} \mathrm{BM}$ neutrophil isolation

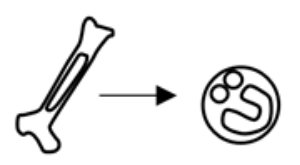

B

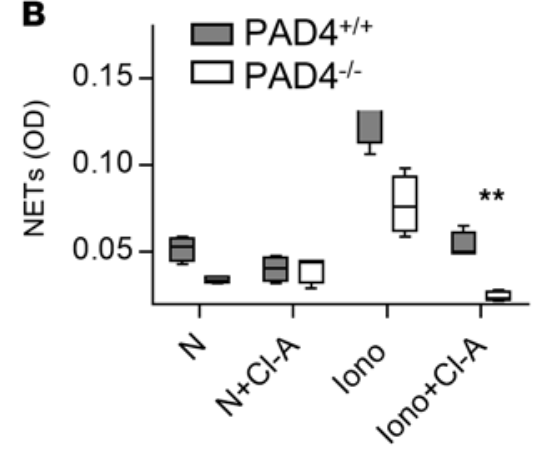

C

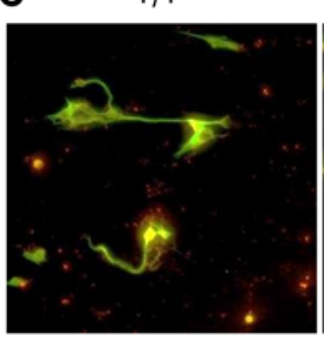

$-/-$

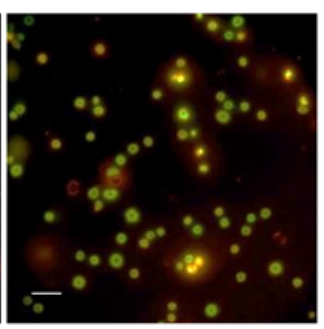

D

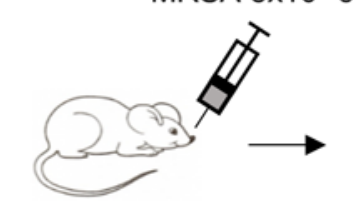

$\mathrm{PAD}^{+/+}, \mathrm{PAD}^{+/-}$or PAD4 - mice
E

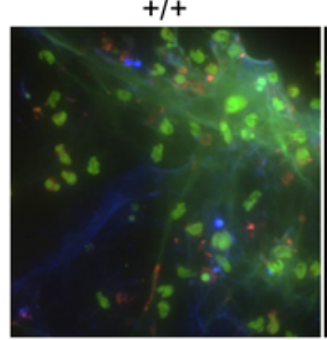

$-/-$

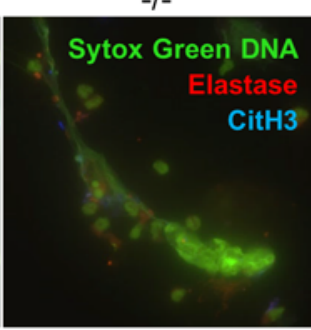

$+/-$

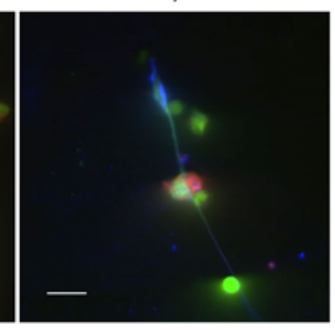

I
F
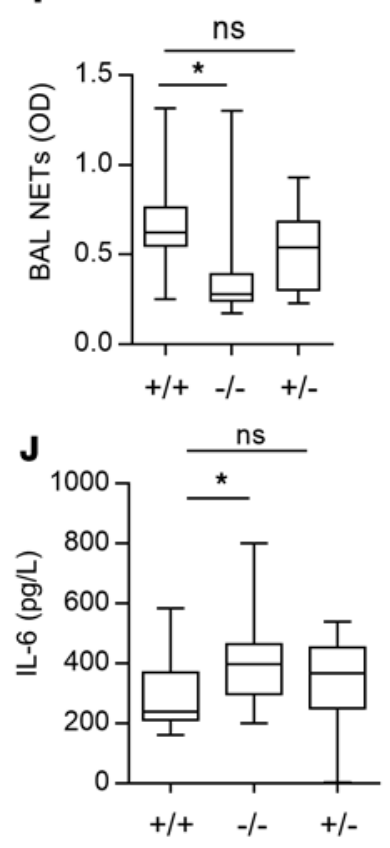

BAL

Blood

Lung
G
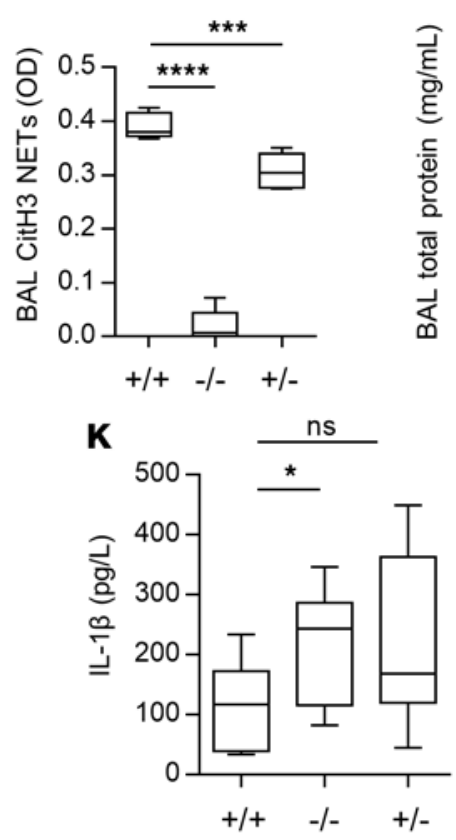

H

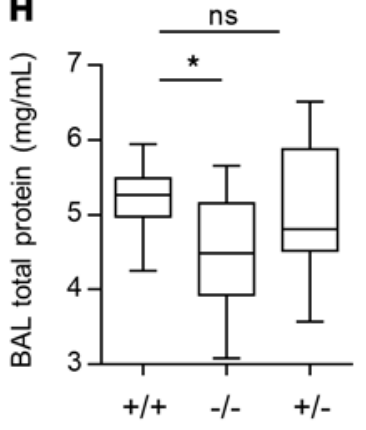

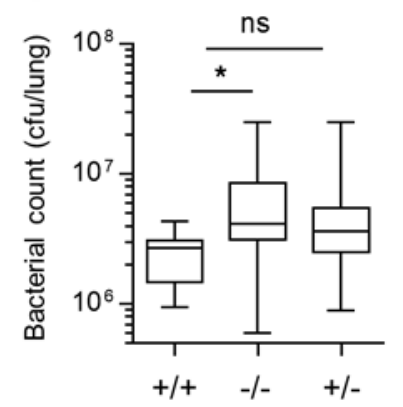

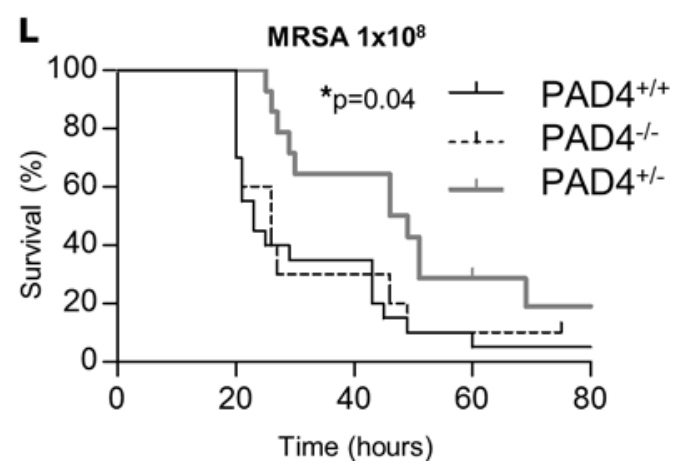

Figure 3. Lung injury, bacterial counts, and survival in NETosis-impaired mice. (A-C) Bone marrow neutrophils were isolated from WT or PAD4-/- mice, pretreated with Cl-amidine (200 $\mu \mathrm{M})$, and stimulated in vitro with ionomycin ( $4 \mu \mathrm{M})$ for 4 hours. (B) Neutrophil extracellular traps (NETs) in neutrophil supernatants were quantified by neutrophil elastase-DNA (NE-DNA) ELISA ( $n=4)$ and (C) visualized by immunofluorescence (DNA, green; NE, red). Scale bar: 20 m. (D-K) WT, PAD4 ${ }^{-/}$, and PAD4+/- littermates were challenged in vivo with methicillin-resistant Staphylococcus aureus (MRSA; $5 \times 10^{7}$ CFU, i.t.). (E) Bronchoalveolar lavage (BAL) was fixed ex vivo and visualized by immunofluorescence (DNA, green; NE, red; citrullinated histone H3 [CitH3], blue). Scale bar: $20 \mu \mathrm{m}$. BAL, blood, and lung were collected at 24 hours. (F) NETs (NE-DNA ELISA), (G) CitH3-DNA complexes, and (H) protein content were quantified in BAL. (I) Bacterial counts in the lung. ( $\mathbf{J}$ and $\mathbf{K}) \mathrm{IL}-6$ and IL-1 $\beta$ concentration in BAL. (B, F-K) Data were analyzed using $1-$ way ANOVA ( $n=11-19) .{ }^{*} P \leq 0.05$, ${ }^{* *} P \leq 0.01,{ }^{* *} P \leq$ $0.001,{ }^{* * *} P \leq 0.0001$. (L) Survival curves for WT, $P A D 4^{-/-}$, and $P A D 4^{+/-}$littermates challenged with MRSA ( $1 \times 10^{8} \mathrm{CFU}$, i.t.). Survival curves were compared using Gehan-Breslow-Wilcoxon test $(n=10-20)$. ns, not significant.

care unit (ICU) and monitors these patients for 5 days for the development of ARDS (29). The demographics of our cohort are listed in Supplemental Table 1. Using our NE-DNA ELISA on plasma samples, we found that plasma NETs were higher in patients with clinical infection-related ARDS (pneumonia and nonpulmonary sepsis) compared with patients admitted with acute cardiac conditions (our 
A

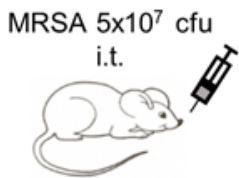

WT mice
DNasel i.t.

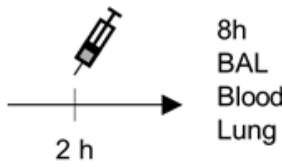

D

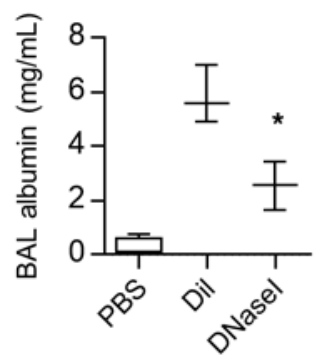

I

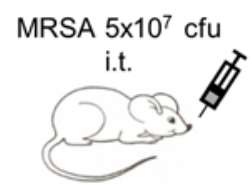

WT mice
E

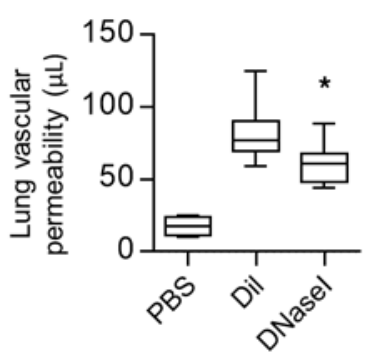

B

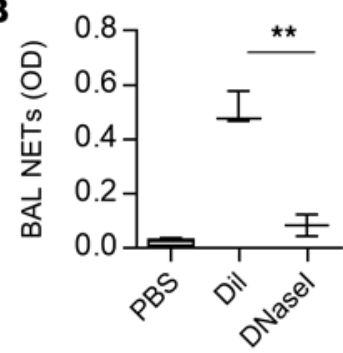

C

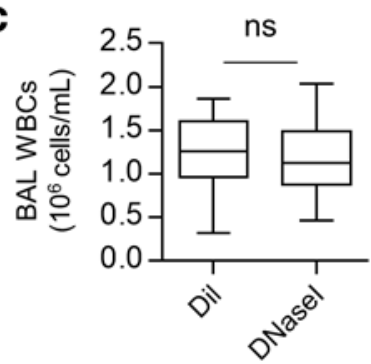

$\mathbf{F}$

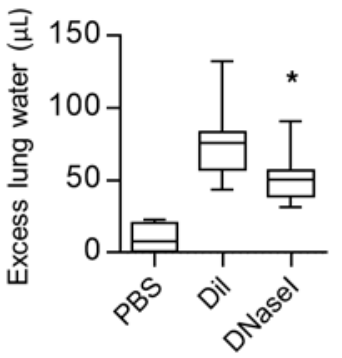

G

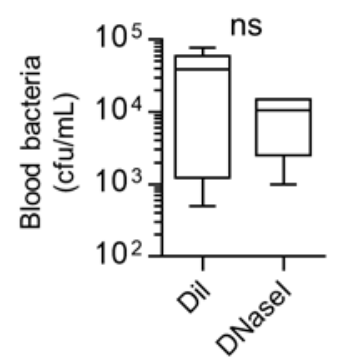

H

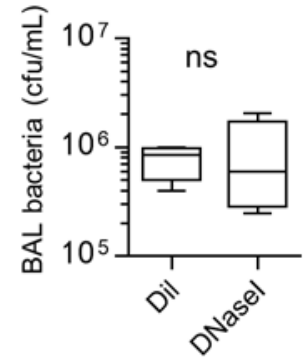

\section{J}

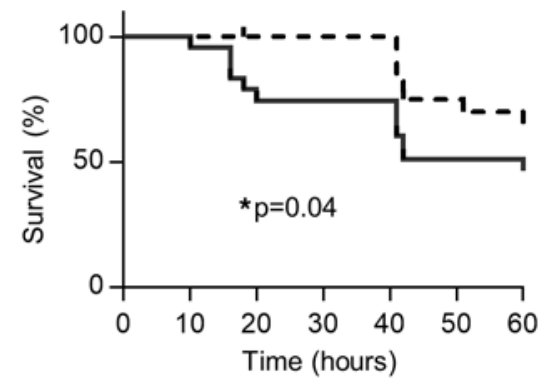

- - DNasel $(n=24)$

Diluent $(n=24)$

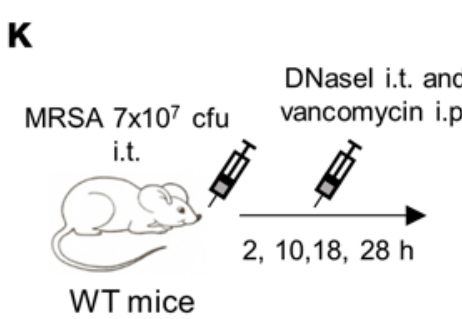

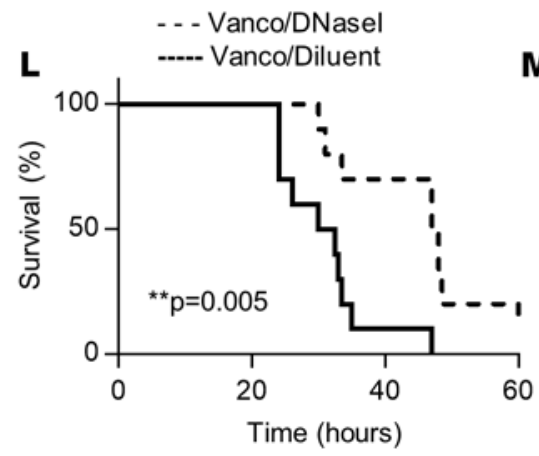
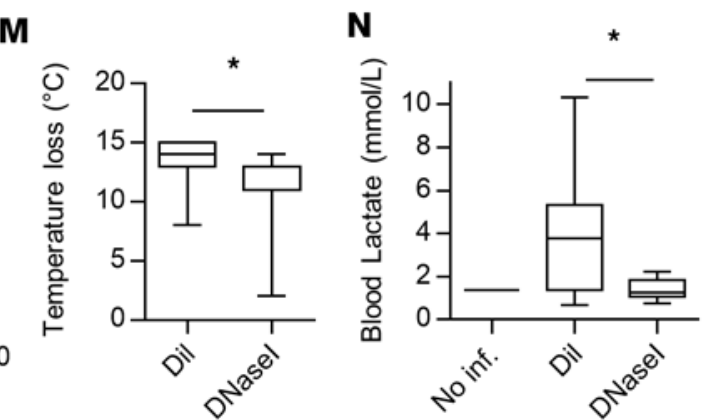

Figure 4. Degradation of NETs with DNase I reduces lung injury and improves survival. (A-H) WT mice were challenged with methicillin-resistant Staphylococcus aureus (MRSA; $5 \times 10^{7} \mathrm{CFU}$, i.t.) and treated 2 hours later with DNase I (2,000 units, i.t.) to disrupt neutrophil extracellular traps (NETs). Bronchoalveolar lavage (BAL), blood, and lung tissue were collected 8 hours after infection. (B) BAL NETs (neutrophil elastase-DNA complexes), (C) BAL WBC, (D) BAL albumin concentration, (E) lung vascular permeability to albumin, (F) excess lung water, (C) bacterial counts in blood, and (H) BAL bacterial counts were quantified. (I and J) Survival experiment schema and curves for mice challenged with MRSA ( $5 \times 10^{7} \mathrm{CFU}$, i.t.) and treated with DNase I or diluent control (i.t.) at 2, 10, 18, and 28 hours after infection $(n=24)$. (K-N) Mice were challenged with MRSA $\left(7 \times 10^{7} \mathrm{CFU}\right.$, i.t.) and treated with vancomycin (150 mg/kg, i.p.) and DNase I (2,000-4,000 units, i.t.) or diluent control at 2, 10, 18, and 28 hours after infection $(n=10)$. (M) Body temperature loss and (N) blood lactate 24 hours after infection. (J and $\mathbf{L})$ Survival curves were compared using Gehan-Breslow-Wilcoxon test. (B-H, M, N) Data were analyzed using Student's $t$ test $(n=3-10) .{ }^{*} P \leq 0.05,{ }^{* *} P \leq 0.01$. ns, not significant.

control group, Figure 6A). Among patients with microbiologically confirmed pneumonia, those with ARDS had higher plasma NETs compared with patients who never developed ARDS (Figure 6B), and there was a trend towards higher plasma NETs in sepsis patients with concomitant ARDS (Figure 6C). NETs in the plasma increased with the severity of ARDS (Figure 6D) and were associated with ARDS mortality (Figure 6E) and also mortality in patients with sepsis or pneumonia ( \pm ARDS) (Figure 6F). 
A

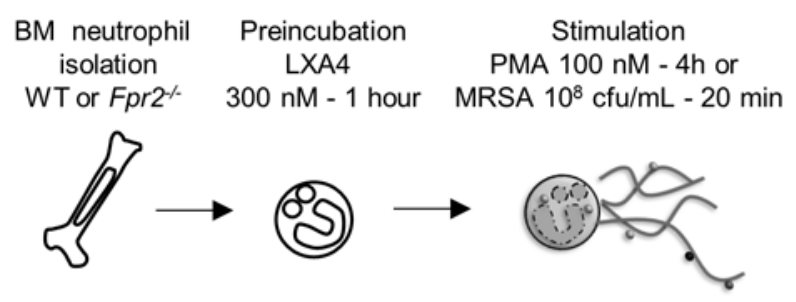

C

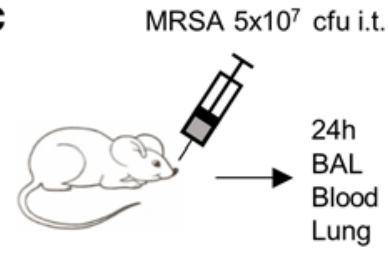

WT or Fpr2 mice

G

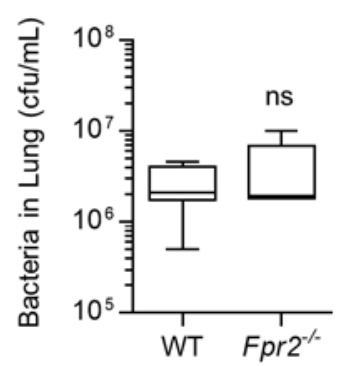

H

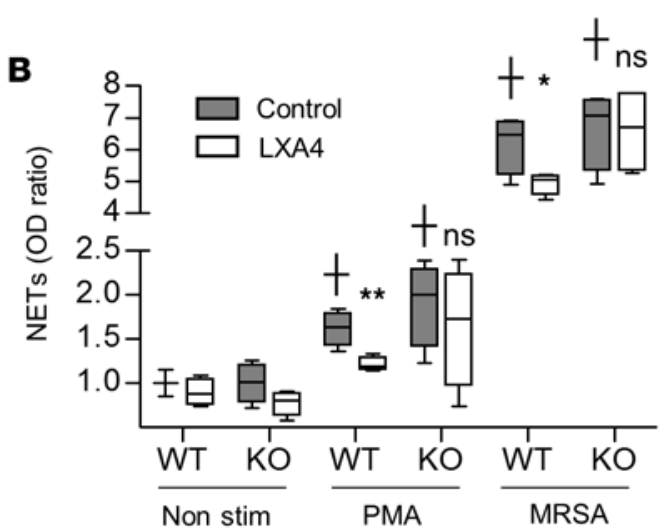

E

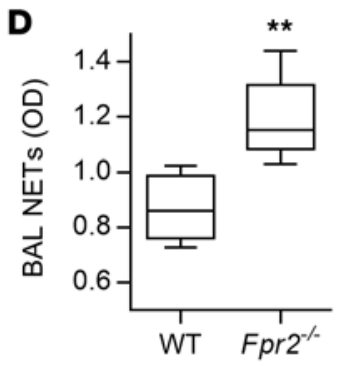

E
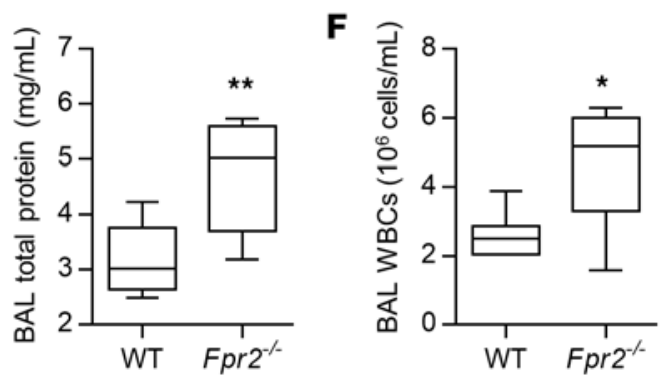

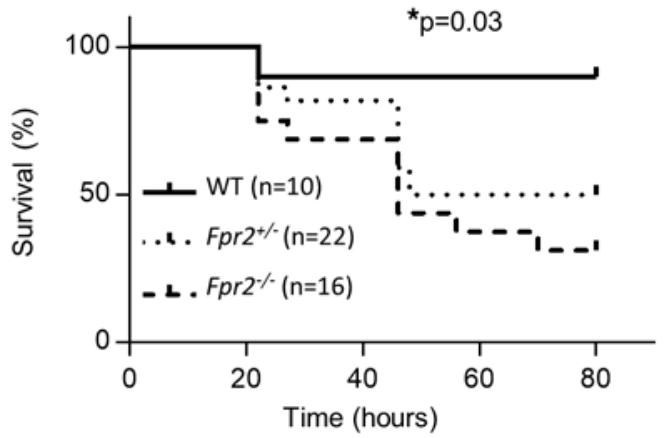

Figure 5. Lipoxin pathway regulates NET production. (A and B) Bone marrow neutrophils were isolated from WT or Fpr $2^{-/-}$mice, preincubated 1 hour with $300 \mathrm{nM}$ lipoxin $\mathrm{A}_{4}$ (LXA4), and stimulated in vitro with $100 \mathrm{nM}$ PMA or $10^{8} \mathrm{CFU} / \mathrm{ml}$ methicillin-resistant Staphylococcus aureus (MRSA). (B) Neutrophil extracellular traps (NETs) in neutrophil supernatant were quantified by ELISA (neutrophil elastase-DNA [NE-DNA] complexes) $(n=4)$. PMA and MRSA increase NET production over nonstimulated neutrophils $\left({ }^{\dagger} P \leq 0.01\right)$ and LXA4 inhibits NET production compared with WT neutrophils treated with control $(*)$. LXA4 did not reduce production in Fpr2 ${ }^{-/-}$neutrophils. (C-C) WT, $\mathrm{Fpr}^{+/-}$, or $\mathrm{Fpr2}^{-/-}$littermates were challenged in vivo with MRSA (5 $\times 10^{7} \mathrm{CFU}$, i.t.). Bronchoalveolar lavage (BAL), blood, and lung tissue were collected at 24 hours. (D) BAL NETs (NE-DNA complexes), (E) BAL total protein, (F) BAL WBC, and (G) lung bacterial counts were quantified. Data were analyzed using Student's $t$ test $(n=5-6) .{ }^{*} P \leq 0.05,{ }^{* *} P \leq 0.01$. (H) Survival curves for WT, Fpr $2^{+/-}$, or Fpr2 ${ }^{-/-}$littermates challenged with MRSA ( $5 \times 10^{7} \mathrm{CFU}$, i.t.). Survival curves were analyzed using Gehan-Breslow-Wilcoxon test $(n=10-22)$. ns, not significant.

DNase I plasma levels are associated with sepsis-induced ARDS. We hypothesized that the association of higher plasma NETs with ARDS severity and mortality is a result of ineffective NET clearance by plasma DNases. DNase I is the major endonuclease found in the blood (30) and recent studies have shown that patients with low levels of DNase I have higher amounts of NETs and anti-nucleosome antibodies $(11,31,32)$. However, the role of DNase I activity in ARDS has not been tested. We observed that DNase I levels were higher in ARDS patients than in cardiac controls (Supplemental Figure 4A). We did not find a difference in DNase I levels in patients with pneumonia with or without ARDS (Supplemental Figure 4B and Figure 7A), and DNase I level was not associated with ARDS severity or mortality (Supplemental Figure 4, D-F). However, in the group with nonpulmonary sepsis, patients with ARDS had a trend toward lower DNase I levels compared with patients without ARDS (Supplemental Figure 4C). We also assessed NET/DNase I ratios to test the hypothesis that a higher ratio would associate with ARDS and worse ARDS outcomes. The NET/DNase I ration was higher in ARDS patients compared with cardiac controls, was higher in pneumonia or sepsis patients with 

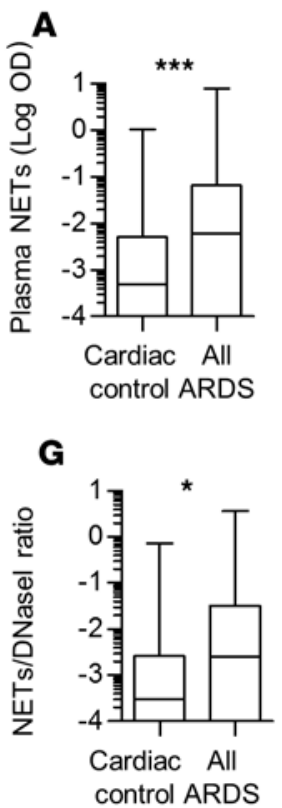

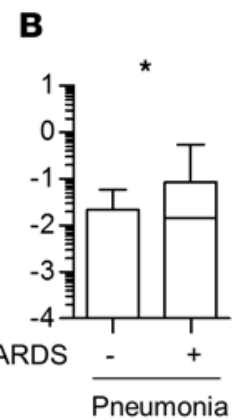

H

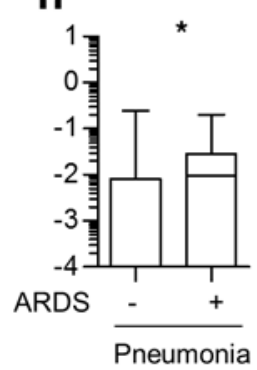

C

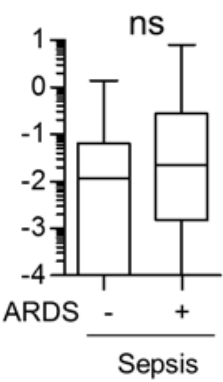

I

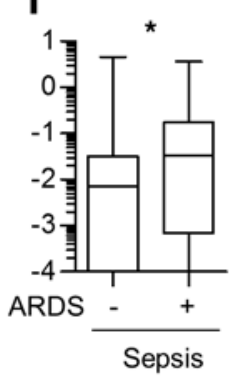

D
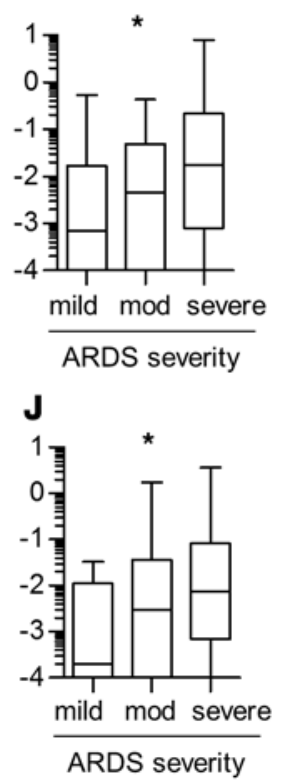

E

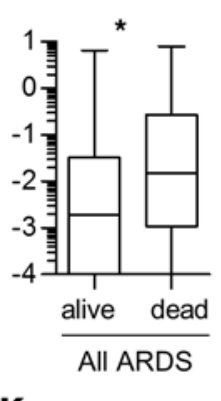

K

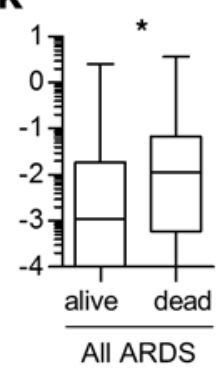

$\mathbf{F}$

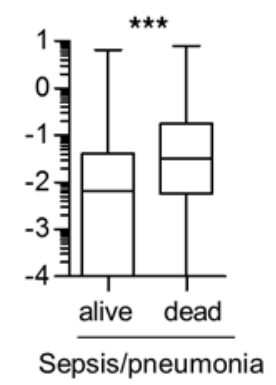

$\mathbf{L}$

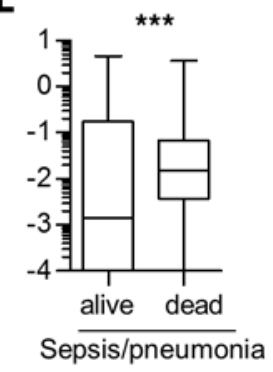

Figure 6. NETs are present in ARDS patients with infection. NETs and NET/DNase I ratio correlate with ARDS severity and mortality. (A-F) Plasma neutrophil extracellular traps (NETs) (neutrophil elastase-DNA complexes) and (G-L) plasma NET/DNase I ratio from patients with (A and $\mathbf{G})$ acute respiratory distress syndrome (ARDS) $(n=104)$ or with acute cardiac conditions $(n=40), P=0.0008, P=0.02$; $(\mathbf{B}$ and $\mathbf{H})$ pneumonia with and without ARDS ( $n=24$ and 14 , respectively), $P=0.03, P=0.03$; (C and I) nonpulmonary sepsis with and without ARDS ( $n=73$ and 21, respectively), $P=0.09, P=0.03$; or ( $D$ and $\mathbf{~ ) ~ m i l d , ~ m o d - ~}$ erate, or severe ARDS according to the Berlin definition $(n=19,30$, or 25 , respectively), $P=0.02, P=0.05$. (E and K) Association of NETs with ARDS mortality $(n=64,40), P=0.03, P=0.04$ and $(\mathbf{F}$ and $\mathbf{L})$ mortality in sepsis/pneumonia $(n=102,47), P=0.001, P=0.0003$. Data were analyzed using the Mann-WhitneyWilcoxon test. ns, not significant.

ARDS, and the ratio was associated with ARDS severity and mortality (Figure 6, G-L). Importantly, we observed that a low DNase I plasma concentration in sepsis patients on initial presentation was associated with the subsequent development of ARDS (Figure 7B).

\section{Discussion}

In response to pathogen challenge, a delicate balance exists between an immune response that adequately controls the infection but is not so exuberant that it results in collateral damage to host tissues (33). In the case of ARDS, it has long been appreciated that a cardinal feature of the syndrome is an early, robust neutrophilic response in both sterile and pathogen-induced etiologies (4). An ideal therapeutic, of which none exists for ARDS, would harness the tissue-injuring effects of the neutrophil while leaving the microbe-clearing capacities relatively unaffected. Here, we demonstrate that maintaining the correct balance of NET formation in tissues is essential for preserving the power of NETs for microbial containment while minimizing damage to the host.

We unequivocally demonstrate by independent methodologies (ELISA, immunofluorescence, intravital lung microscopy) that NETs are a cardinal feature of lung injury after bacterial challenge in mice. Our technique to study NET formation ex vivo in neutrophils recovered from the alveolar spaces is a technical advance in studying a primed population of cells (34) for NET formation, and overcomes the limitations and challenges of studying bone marrow neutrophils that are immature and may be difficult to stimulate. NETs accumulate relatively early after pathogen challenge in both alveolar and vascular spaces in the lung and correlate well with lung permeability changes, which is the cardinal feature of ARDS. The elaboration of NETs in the lung vasculature observed by intravital microscopy is a dynamic process of release and degradation, which was a clue to their regulation that informed our therapeutic approaches and human investigations.

Our therapeutic studies involving PAD4 inhibition and DNase I cleavage of NETs have informed a concept that NET balance and the correct timing of NET inhibition is critical to efficient microbial containment while limiting host damage. For example, complete deficiency of PAD4 produced mixed results in the injury models, with modest reduction in lung injury but at the expense of decreased bacterial clearance. A partial deficiency 

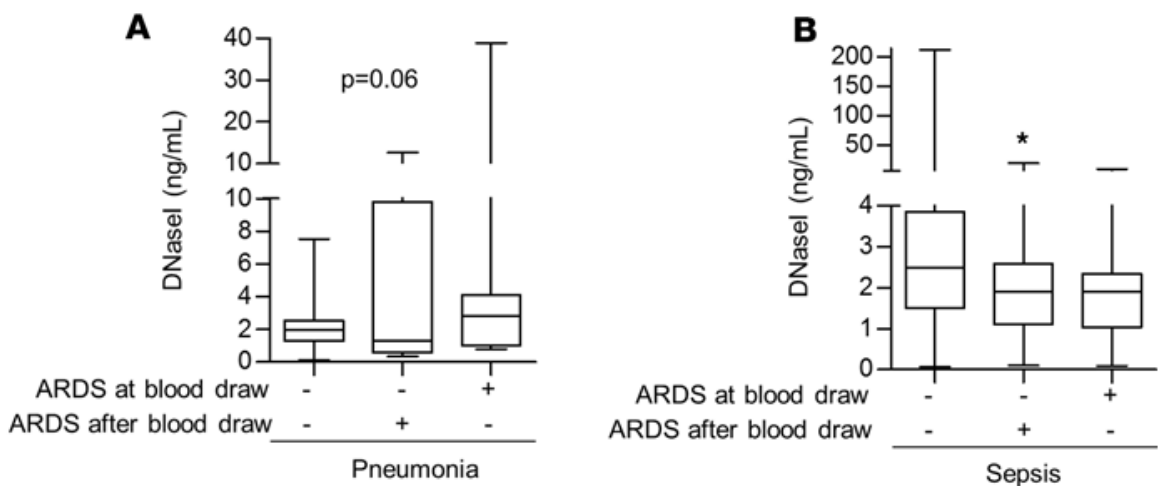

Figure 7. DNase I plasma levels are associated with ARDS development in patients with nonpulmonary sepsis. (A) DNase I concentration from patients with pneumonia who never developed acute respiratory distress syndrome (ARDS) $(n=16)$, who developed ARDS after blood collection $(n=4)$, or with ARDS at time of blood collection $(n=20), P=0.06$. (B) DNase I concentration from patients with nonpulmonary sepsis who never developed ARDS $(n=83)$, who developed ARDS after blood collection $(n=15)$, or with ARDS at time of blood collection $(n=21)$, ${ }^{*} P=0.04$. Data were analyzed using the Mann-Whitney-Wilcoxon test.

in PAD4 (heterozygous mice) produced a surprising survival advantage, perhaps from a more balanced production of NETs sufficient for microbial containment, but not so exuberant as to produce lung injury. Similarly, the survival advantage in DNase I-treated animals likely resulted from the reduction in NETs (but not complete absence), which produced salutary effects on reducing lung injury without adversely affecting bacterial load. Notably, these results lend themselves to clinical translation and implementation, since NETs could be targeted after infection onset (with PAD inhibitors or DNase I) without the need for their complete abolition. Targeting NETs after ARDS onset or even during the resolution phase is a practical approach, since it is difficult to predict the onset of ARDS and implement early therapies. Our therapeutic approach with DNase I also paired favorably with pathogen-specific antibiotics, which also argues for clinical relevancy. Finally, the survival advantage in the DNase I-treated group may be an underestimate, since we stopped treatment at 28 hours after infection.

The residual presence of NETs in $P A D 4^{-/}$and in $\mathrm{Cl}$-amidine-treated mice after infection indicates that citrullination is not required for NET production in vivo. It confirms in vitro data indicating that after different stimuli, citrullination of histone $\mathrm{H} 3$ occurs during NETosis but is not required for NET induction (35). Inhibiting citrullination may still be useful to decrease NET pathogenicity, as $\mathrm{Cl}$-amidine-treated mice have significantly less inflammation. These effects of $\mathrm{Cl}$-amidine may be explained by the decrease in plasma NETs (Supplemental Figure 3D) and the role of NETs in regulating inflammatory cytokines directly or indirectly by modulating other immune cells $(36,37)$. Moreover, inhibiting PAD4 can have non-NET-associated effects. Histone citrullination could change extracellular histone pathogenicity or immunogenicity by modifying its binding with cell surfaces or receptors (38). PAD4 also has other targets such as the antimicrobial peptide LL-37, which has an impaired antibacterial activity against MRSA when citrullinated (39), and PAD4 can also regulate gene expression by changing chromatin structure in promoter regions (40-42).

We have also identified a pathway that is a potentially novel regulator of NET formation - the antiinflammatory, proresolving lipoxin pathway. Lipoxins are endogenous lipid mediators produced in response to inflammation that have potent effects on immune cells, including neutrophils (43). However, to our knowledge there have been no previous studies investigating the effects of lipoxins on NETosis. The Fpr2 agonist, lipoxin $\mathrm{A}_{4}$, reduced NET formation in an in vitro NET assay, and mice deficient in Fpr2 had excessive NET formation after bacterial challenge that was associated with more lung injury and excess mortality. Fpr2 ligands modify calcium flux in neutrophils, which is involved in NETosis-related pathways such as citrullination and ROS production, and they promote neutrophil apoptosis (44-46), a known antagonist pathway for NETosis (47). These results open the door for new investigation on the mechanisms responsible for these effects and the therapeutic potential of Fpr2 agonists in regulating NET formation in ARDS.

We extended our studies to humans with pneumonia or nonpulmonary sepsis etiologies of ARDS using a potentially novel human cohort, studying the early stages of critical illness. We focused on pneumonia and nonpulmonary sepsis as causes of ARDS because they are most relevant to our mouse studies and are also the 2 leading causes of ARDS in most studies, accounting for more than $75 \%$ of cases (48). Using our NET 
ELISA in plasma samples collected on initial presentation, we found that NETs are increased in patients with ARDS compared with critically ill controls, and plasma NETs are higher in pneumonia patients who eventually develop ARDS compared with pneumonia patients who do not develop ARDS. Importantly, NETs also increase with ARDS severity and are strongly associated with mortality, which points to their biological potency and potential for therapeutic intervention. Patients who overproduce NETs may fit into the hyperinflammatory subphenotype of ARDS that is associated with worse clinical outcomes (49).

Informed by our intravital microscopy studies and results from the DNase I experiments in mice, we next tested the association of DNase I plasma levels with progression to ARDS. We hypothesized that lower DNase I plasma levels would be paralleled by higher plasma NETs (11), which would be barrier disruptive. Supporting this hypothesis, recent experimental data indicate that a deficiency in endogenous DNases produces an impairment in NET degradation leading to vascular occlusion and organ damage in sepsis (12). Indeed, DNase I levels were higher in sepsis patients who never developed ARDS compared with those who presented with or eventually developed ARDS. These results do not conclusively prove that higher DNase I plasma levels are protective in ARDS, but the results do fit overall with the hypothesis that a critical balance of NETs is necessary to prevent lung injury and to maintain microbial control. We found value in expressing NETs and DNase I as a ratio, which was also strongly associated with the development of ARDS and ARDS severity/mortality. The association of DNase I plasma level and ARDS development was stronger in sepsis patients than in pneumonia patients, perhaps because in pneumonia patients, NETs may be partially contained in the lung and it would be more revealing to test BAL NET and DNase I levels.

There are limitations to our study. Although we believe that our intravital microscopy images are the first to show the dynamic production of NETs in the lung, we did not colocalize a neutrophil-specific marker to the extracellular DNA. However, the morphology of the extracellular DNA fibers is consistent with NETs, and we do show that neutrophils are the source of the intravascular NETs and intensely colocalize to areas of alveolar NETs. We did not test the i.v. delivery of DNase I, since our experimental results (BAL NET ELISA, lung intravital imaging) point to NETs in the alveolar spaces as being the predominant pattern. However, we have successfully used i.v. DNase I in an indirect model of lung injury (TRALI) and ultimately the inciting cause of lung injury may dictate the best delivery route. Our human studies only associate higher plasma NETs with ARDS severity and mortality, but do not prove causation.

In conclusion, the elaboration of NETs is a cardinal feature of both experimental acute lung injury and ARDS. NETs tightly correlate with the development of lung barrier injury and strategies to reduce NET levels (but not completely abolish) have favorable effects on lung function without compromising bacterial containment. In the modern era of antibiotic treatment, decreasing NETs may be a reasonable strategy to harness the powerful potential of the neutrophil to combat microbial challenges. Indeed, anti-NET therapy could be developed as an adjunctive treatment to antibiotics and supportive care for pneumonia and sepsis.

\section{Methods}

Mice. Mice were housed and bred under specific pathogen-free conditions at the UCSF Laboratory Animal Research Center, and all experiments conformed to ethical principles and guidelines approved by the UCSF Institutional Animal Care and Use Committee. We used 8- to 12-week-old male mice for the experimental procedures. C57BL/6, $m T m G$, and $n T n G$ mice were purchased from the Jackson Laboratories. LysM-GFP and Fpr2/3--- were used as previously described (23). MRP8-Cre mice were obtained from Clifford Lowell (UCSF), and $P A D 4^{-1-}$ mice were obtained from Kerri Mowen (The Scripps Research Institute, La Jolla, California, USA). To track neutrophils using intravital microscopy, MRP8-Cre-expressing mice were crossed with $m \operatorname{Tm} G$ or $n T n G$ reporter strains.

MRSA mouse model. We used the SF8300 strain of MRSA, a minimally passaged USA300 clinical strain representative of the epidemic clone USA300-0114 obtained from collaborators at UCSF (22). Stock solutions at the mid-logarithmic growth phase $\left(10^{10} \mathrm{CFU} / \mathrm{ml}\right)$ were aliquoted and frozen at $-80^{\circ} \mathrm{C}$ using standard techniques (22). On the day of the experiment, a vial of SF8300 was thawed and diluted with PBS to the concentration needed for i.t. instillation into anesthetized mice using our direct visual instillation method (50). For MRSA-induced pneumonia, we used $5 \times 10^{7} \mathrm{CFU} /$ mouse and mice were sacrificed at 6 , $10,14,18$, or 24 hours after instillation. For survival experiments, $1 \times 10^{8} \mathrm{CFU} /$ mouse was instilled i.t. In selected experiments, we treated mice with recombinant DNase I (Roche; 2,000-4,000 units/mouse, i.t.) every 8-12 hours, vancomycin $(150 \mathrm{mg} / \mathrm{kg}$, i.p.) every 8-12 hours until the end of the experiment, and $\mathrm{Cl}-$ amidine (Calbiochem, 506282, $50 \mathrm{mg} / \mathrm{kg}$, i.p.) 3 hours after infection. 
$P$. aeruginosa mouse model. PAO1 (ATCC 15692) is the most commonly used strain of $P$. aeruginosa for laboratory research and was obtained from collaborators at UCSF (51). We used PAO1 in a 24-hour model of pneumonia/ALI by instilling $1 \times 10^{7}$ to $5 \times 10^{7} \mathrm{CFU} /$ mouse i.t.

Intratracheal infection and lung injury model. BAL was obtained at 24 hours by inserting a 20-gauge catheter into the trachea through which $1 \mathrm{ml}$ of cold PBS was flushed back and forth 3 times. Total protein concentration in the cell-free BAL was determined using a BCA protein assay kit (Thermo Fisher Scientific). BAL leukocytes were quantified using a Coulter counter (Beckman Coulter), and the cellular differential was determined by cytospin preparation (Cytospin 3; Thermo Electron Corp) and Diff-Quick staining. In selected experiments where mice were treated i.t. with protein (DNase I treatment), we quantified albumin concentration in BAL fluid by ELISA (Bethyl Laboratories) instead of BCA total protein. Bacterial counts were determined for blood, BAL, or lung homogenate plated on TSA-5\% sheep blood agar plates (Teknova) by colony numeration. For survival experiments, body weight and rectal temperature were monitored. Blood lactate was measured in arterial blood collected from the left ventricle using a blood gas machine (VetScan, Abaxis) and CG4+ cartridges.

Cytokine measurements. TNF- $\alpha$, IL-1 $\beta$, IL-10, IL-12, IFN- $\gamma$, MCP-1, and IL-6 concentrations were quantified in BAL fluid from infected mice using the Mouse Inflammation Multiplex Immunoassay Kit (Abcam, ab213396).

Lung edema and vascular permeability measurements. We quantified pulmonary edema by measuring bloodless, extravascular lung water as previously described (52). Lungs were removed at the end of the experiment, weighed, homogenized, and placed in a drying oven for 24 hours. Measuring hemoglobin in the lung homogenate allows for the calculation of bloodless extravascular lung water. We measured lung vascular protein permeability by treating mice with i.p. ${ }^{125} \mathrm{I}$-labeled albumin (Iso-Tex Diagnostics). The ratio of the radioactivity measured with a gamma counter in the blood and the bloodless lung was used to calculate the lung extravascular plasma equivalents $(\mu 1)(52)$. Blood was removed by cardiac puncture and analyzed on a mouse complete blood count instrument (Genesis, Oxford Science) and stored as plasma at $-80^{\circ} \mathrm{C}$.

NET ELISA. We have developed a custom ELISA to quantify soluble NET components in cell culture supernatants, BAL, and plasma from mice or human samples (19). Antibodies against neutrophil elastase M-18 (sc-9521) or G2 (sc-55549) for mouse, C17 (sc-9520) for human (all Santa Cruz Biotechnology), or citrullinated histone H3 (Abcam, ab5103) were used for capture, and an anti-DNA-HRP conjugate (Cell Death Detection ELISA ${ }^{\text {plus }}$ Kit, Roche) was used as the detection antibody.

NET in vitro experiments. Bone marrow cells were harvested in room temperature PBS without calcium and layered over a Percoll gradient $(80 \%, 65 \%, 50 \%)$. After centrifugation (1,000 $g$ without braking, 30 minutes, room temperature), neutrophils were recovered at the 50\%/65\% interface. Purity was checked by cytospin and was greater than $90 \%$ in all experiments. Red blood cells were eliminated after incubation of the pellet with $3 \mathrm{ml}$ of ice-cold $\mathrm{H}_{2} 0$ for 30 seconds and $1 \mathrm{ml}$ of $3.6 \mathrm{M} \mathrm{NaCl}$. Cells were resuspended in RMPI with $1 \%$ serum, and $5 \times 10^{5}$ cells in $100 \mu 1$ were plated per well in 96-well plates. Neutrophils were cultured at $37^{\circ} \mathrm{C}$ and $5 \% \mathrm{CO}_{2}$ and stimulated with either $4 \mu \mathrm{M}$ ionomycin (Adipogen) for 4 hours, 100 nM PMA (Sigma-Aldrich) for 4 hours, or MRSA $\left(10^{8} \mathrm{CFU} / \mathrm{ml}\right)$ for 20 minutes. In selected experiments, neutrophils were pretreated for 1 hour with Cl-amidine $(200 \mu \mathrm{M})$ or lipoxin $\mathrm{A}_{4}$ (EMD Millipore, $300 \mathrm{nM}$ ).

NET immunofluorescence. For ex vivo NET immunofluorescence, BAL from MRSA- or PAO1-infected mice, or from MIP-2-treated mice (i.t., R\&D Systems, 452-M2), was settled 1 hour in 12-well chambers (Ibidi, 81201$)$ at $37^{\circ} \mathrm{C}$ and $5 \% \mathrm{CO}_{2}$. For in vitro NETs, we plated $5 \times 10^{5}$ neutrophils $/ 200 \mu 1\left(2.5 \times 10^{5}\right.$ cells $/$ $\mathrm{ml}$ ) and stimulated the cells for 3 hours at $37^{\circ} \mathrm{C}$ and $5 \% \mathrm{CO}_{2}$. For ex vivo or in vitro NETs experiments, cells were fixed with paraformaldehyde, then incubated for 1 hour at room temperature with PBS/3\% BSA, and stained at $4^{\circ} \mathrm{C}$ overnight with goat anti-neutrophil elastase M-18 or G2 (sc-9521 or sc-55549, 1:200), rabbit anti-citrullinated histone H3 (ab5103, 1:500), or histone H2B (Abcam, ab52599, 1:500). Secondary antibodies were added for 1 hour at room temperature and SYTOX Green for 10 minutes at room temperature (Invitrogen, S7020, 1:10,000). Images were captured using a Nikon Ti microscope.

Lung intravital microscopy. A modified version (53) of the previously published method of stabilized lung imaging (54) was used. Mice were anesthetized with ketamine and xylazine and secured with tape to a custom heated microscope stage. A small tracheal cannula was inserted, sutured into place, and attached to a MiniVent mouse ventilator (Harvard Apparatus). Mice were ventilated with a tidal volume of $10 \mu 1$ of compressed air $\left(21 \% \mathrm{O}_{2}\right)$ per gram of mouse weight, a respiratory rate of $130-140$ breaths per minute, and a positive-end expiratory pressure of $2-3 \mathrm{cmH}_{2} \mathrm{O}$. Isoflurane was continuously delivered to maintain anesthe- 
sia and mice were administered $300 \mu 1$ of $0.9 \%$ saline solution i.p. every hour. Mice were placed in the right lateral decubitus position and a small surgical incision was made to expose the rib cage. A second incision was then made into the intercostal space between ribs 4 and 5, through the parietal pleura, to expose the surface of the left lung lobe. A flanged thoracic window with an 8-mm coverslip was inserted between the ribs and secured to the stage using a set of optical posts and a $90^{\circ}$ angle post clamp (Thor Labs). Suction (20-25 $\mathrm{mmHg}$ ) was applied (Amvex Corporation) to gently immobilize the lung. The 2-photon microscope objective was then lowered into place over the thoracic window. Intravital imaging was performed using a Nikon A1R multi-photon microscope equipped with a Mai Tai DeepSee IR Laser (Spectra Physics) (UCSF Biological Imaging Development Core). We captured a $0.4-\mathrm{mm}^{2} x-y$ surface area at $40-\mu \mathrm{m} z$ depth, capturing a complete image every 1 minute for 120 minutes. When SYTOX Green was used with LysM-eGFP mice, eGFP ${ }^{+}$neutrophils were detected with the blue PMT detectors and SYTOX Green DNA with the green PMT detectors, as eGFP has a lower emission peak (509 nm) than SYTOX Green (523 nm). Images were analyzed using Imaris 7.6.1 software (Bitplane).

EARLI human plasma samples. The emphasis of the EARLI study is the collection of clinical data and biological samples in the emergency department at a time that often precedes the development of ARDS. The primary inclusion criterion is admission to an ICU from the emergency department; exclusion criteria include isolated neurologic or neurosurgical diagnoses and trauma. Patients are phenotyped for sepsis, pneumonia, and other diagnoses by physician-investigators blinded to the results of biological analyses on patient samples. Patients are monitored for 5 days for ARDS, which is determined by 2-physician review of all chest radiographs and clinical data during that time period, and for 60-day in-hospital mortality. We used the American-European Consensus Conference (AECC) definition of ARDS, since this definition allows inclusion of patients who are not mechanically ventilated (55). We also used the Berlin definition of ARDS in analyses of ARDS severity (56). We included a potentially novel control group - patients admitted with acute cardiac conditions who may have cardiogenic pulmonary edema but should not have permeability pulmonary edema. We have used the EARLI cardiac patients in a previous study that confirmed that NETs are negligible in this group (17). Plasma samples were obtained as soon as possible after enrollment but no later than 24 hours after ICU admission. In terms of timing relative to ARDS, some patients already had ARDS when these samples were collected (ARDS at blood draw), while in other cases, sample collection predated ARDS development (ARDS after blood draw); some patients never developed ARDS (at-risk controls, cardiac controls). Samples were collected in EDTA tubes, processed immediately after collection, and stored at $-80^{\circ} \mathrm{C}$ for batch analysis. NETs were determined using our NE-DNA ELISA, and DNase I concentration was quantified by ELISA (MyBioSource, MBS2024312).

Statistics. All in vivo and in vitro experiments were repeated a minimum of 3 independent times. Unless otherwise noted, all data are presented as min-to-max whiskers and box plots showing the median and interquartile ranges. For the mouse experiments, to determine significance, tests were used as appropriate (2-tailed Student's $t$ test to compare 2 groups, 1-way ANOVA to compare more than 2 groups, GehanBreslow-Wilcoxon test for survival curves; GraphPad PRISM). For the human studies, data were analyzed using the Mann-Whitney-Wilcoxon test. A $P$ value less than or equal to 0.05 was considered significant.

Study approval. All experiments were approved by the Institutional Animal Care and Use Committee and the Committee on Human Research at UCSF. Written informed consent was received from participants prior to inclusion in the study.

\section{Author contributions}

EL, CSC, and MRL designed the research studies. EL and BM conducted experiments. EL acquired the data. EL, BM, HZ, CSC, and MRL analyzed the data. EL and MRL wrote the manuscript.

\section{Acknowledgments}

We thank Kaitlin Corbin, Henry Pinkard, and Adam Fries in the Biological Imaging Development Core at UCSF for microscopy assistance. This work was supported in part by US NIH grants R01 AI125445 and R01 HL130324 (to M.R. Looney) and R01 HL131621 (to C.S. Calfee).

Address correspondence to: Mark R. Looney, 513 Parnassus Avenue, HSE 1355A, San Francisco, California 94143-0130, USA. Phone: 415.476.9563; Email: mark.looney@ucsf.edu. 
1. Ferkol T, Schraufnagel D. The global burden of respiratory disease. Ann Am Thorac Soc. 2014;11(3):404-406.

2. Ashbaugh DG, Bigelow DB, Petty TL, Levine BE. Acute respiratory distress in adults. Lancet. 1967;2(7511):319-323.

3. Thompson BT, Chambers RC, Liu KD. Acute respiratory distress syndrome. N Engl J Med. 2017;377(6):562-572.

4. Matthay MA, Ware LB, Zimmerman GA. The acute respiratory distress syndrome. J Clin Invest. 2012;122(8):2731-2740.

5. Brinkmann V, et al. Neutrophil extracellular traps kill bacteria. Science. 2004;303(5663):1532-1535.

6. Yipp BG, Kubes P. NETosis: how vital is it? Blood. 2013;122(16):2784-2794.

7. Jorch SK, Kubes P. An emerging role for neutrophil extracellular traps in noninfectious disease. Nat Med. 2017;23(3):279-287.

8. Martinod K, et al. PAD4-deficiency does not affect bacteremia in polymicrobial sepsis and ameliorates endotoxemic shock. Blood. 2015;125(12):1948-1956.

9. Kolaczkowska E, et al. Molecular mechanisms of NET formation and degradation revealed by intravital imaging in the liver vasculature. Nat Commun. 2015;6:6673.

10. Beiter K, Wartha F, Albiger B, Normark S, Zychlinsky A, Henriques-Normark B. An endonuclease allows Streptococcus pneumoniae to escape from neutrophil extracellular traps. Curr Biol. 2006;16(4):401-407.

11. Hakkim A, et al. Impairment of neutrophil extracellular trap degradation is associated with lupus nephritis. Proc Natl Acad Sci USA. 2010;107(21):9813-9818.

12. Jiménez-Alcázar M, et al. Host DNases prevent vascular occlusion by neutrophil extracellular traps. Science. 2017;358(6367):1202-1206.

13. Storisteanu DM, et al. Evasion of Neutrophil Extracellular Traps by Respiratory Pathogens. Am J Respir Cell Mol Biol. 2017;56(4):423-431.

14. Papayannopoulos V. Neutrophil extracellular traps in immunity and disease. Nat Rev Immunol. 2018;18(2):134-147.

15. Cheng OZ, Palaniyar N. NET balancing: a problem in inflammatory lung diseases. Front Immunol. $2013 ; 4: 1$.

16. Yost CC, et al. Neonatal NET-inhibitory factor and related peptides inhibit neutrophil extracellular trap formation. J Clin Invest. 2016;126(10):3783-3798.

17. Caudrillier A, et al. Platelets induce neutrophil extracellular traps in transfusion-related acute lung injury. J Clin Invest. 2012;122(7):2661-2671.

18. Rossaint J, et al. Synchronized integrin engagement and chemokine activation is crucial in neutrophil extracellular trap-mediated sterile inflammation. Blood. 2014;123(16):2573-2584.

19. Sayah DM, et al. Neutrophil extracellular traps are pathogenic in primary graft dysfunction after lung transplantation. $A m J$ Respir Crit Care Med. 2015;191(4):455-463.

20. Jones RN. Microbial etiologies of hospital-acquired bacterial pneumonia and ventilator-associated bacterial pneumonia. Clin Infect Dis. 2010;51 Supp1 1:S81-S87.

21. DeLeo FR, Otto M, Kreiswirth BN, Chambers HF. Community-associated meticillin-resistant Staphylococcus aureus. Lancet. 2010;375(9725):1557-1568.

22. Diep BA, et al. Polymorphonuclear leukocytes mediate Staphylococcus aureus Panton-Valentine leukocidin-induced lung inflammation and injury. Proc Natl Acad Sci USA. 2010;107(12):5587-5592.

23. Ortiz-Muñoz G, Mallavia B, Bins A, Headley M, Krummel MF, Looney MR. Aspirin-triggered 15-epi-lipoxin A4 regulates neutrophil-platelet aggregation and attenuates acute lung injury in mice. Blood. 2014;124(17):2625-2634.

24. Lefrançais E, et al. The lung is a site of platelet biogenesis and a reservoir for haematopoietic progenitors. Nature. 2017;544(7648):105-109.

25. Passegué E, Wagner EF, Weissman IL. JunB deficiency leads to a myeloproliferative disorder arising from hematopoietic stem cells. Cell. 2004;119(3):431-443.

26. Hemmers S, Teijaro JR, Arandjelovic S, Mowen KA. PAD4-mediated neutrophil extracellular trap formation is not required for immunity against influenza infection. PLoS ONE. 2011;6(7):e22043

27. Martinod K, et al. PAD4-deficiency does not affect bacteremia in polymicrobial sepsis and ameliorates endotoxemic shock. Blood. 2015;125(12):1948-1956.

28. Kretschmer D, et al. Human formyl peptide receptor 2 senses highly pathogenic Staphylococcus aureus. Cell Host Microbe. 2010;7(6):463-473.

29. Agrawal A, et al. Plasma angiopoietin-2 predicts the onset of acute lung injury in critically ill patients. Am J Respir Crit Care Med 2013;187(7):736-742.

30. Prince WS, Baker DL, Dodge AH, Ahmed AE, Chestnut RW, Sinicropi DV. Pharmacodynamics of recombinant human DNase I in serum. Clin Exp Immunol. 1998;113(2):289-296.

31. Meng W, et al. Deoxyribonuclease is a potential counter regulator of aberrant neutrophil extracellular traps formation after major trauma. Mediators Inflamm. 2012;2012:149560.

32. Martinez-Valle F, Balada E, Ordi-Ros J, Bujan-Rivas S, Sellas-Fernandez A, Vilardell-Tarres M. DNase 1 activity in patients with systemic lupus erythematosus: relationship with epidemiological, clinical, immunological and therapeutical features. Lupus. 2009;18(5):418-423.

33. Kruger P, et al. Neutrophils: Between host defence, immune modulation, and tissue injury. PLoS Pathog. 2015;11(3):e1004651

34. Juss JK, et al. Acute respiratory distress syndrome neutrophils have a distinct phenotype and are resistant to phosphoinositide 3-kinase inhibition. Am J Respir Crit Care Med. 2016;194(8):961-973.

35. Kenny EF, et al. Diverse stimuli engage different neutrophil extracellular trap pathways. Elife. 2017;6:e24437.

36. Duewell P, et al. NLRP3 inflammasomes are required for atherogenesis and activated by cholesterol crystals. Nature. 2010;464(7293):1357-1361.

37. Warnatsch A, Ioannou M, Wang Q, Papayannopoulos V. Inflammation. Neutrophil extracellular traps license macrophages for cytokine production in atherosclerosis. Science. 2015;349(6245):316-320.

38. Chen R, Kang R, Fan XG, Tang D. Release and activity of histone in diseases. Cell Death Dis. 2014;5:e1370

39. Kilsgård $\mathrm{O}$, et al. Peptidylarginine deiminases present in the airways during tobacco smoking and inflammation can citrullinate the host defense peptide LL-37, resulting in altered activities. Am J Respir Cell Mol Biol. 2012;46(2):240-248.

40. Cuthbert GL, et al. Histone deimination antagonizes arginine methylation. Cell. 2004;118(5):545-553. 
41. Li P, et al. Regulation of p53 target gene expression by peptidylarginine deiminase 4. Mol Cell Biol. 2008;28(15):4745-4758

42. Wang Y, et al. Human PAD4 regulates histone arginine methylation levels via demethylimination. Science. 2004;306(5694):279-283.

43. Levy BD, Serhan CN. Resolution of acute inflammation in the lung. Annu Rev Physiol. 2014;76:467-492.

44. Vago JP, et al. Annexin A1 modulates natural and glucocorticoid-induced resolution of inflammation by enhancing neutrophil apoptosis. J Leukoc Biol. 2012;92(2):249-258.

45. E1 Kebir D, et al. 15-epi-lipoxin A4 inhibits myeloperoxidase signaling and enhances resolution of acute lung injury. Am J Respir Crit Care Med. 2009;180(4):311-319.

46. Solito E, Kamal A, Russo-Marie F, Buckingham JC, Marullo S, Perretti M. A novel calcium-dependent proapoptotic effect of annexin 1 on human neutrophils. FASEB J. 2003;17(11):1544-1546.

47. Hakkim A, et al. Activation of the Raf-MEK-ERK pathway is required for neutrophil extracellular trap formation. Nat Chem Biol. 2011;7(2):75-77.

48. Bellani G, et al. Epidemiology, patterns of care, and mortality for patients with acute respiratory distress syndrome in intensive care units in 50 countries. JAMA. 2016;315(8):788-800

49. Calfee CS, et al. Subphenotypes in acute respiratory distress syndrome: latent class analysis of data from two randomised controlled trials. Lancet Respir Med. 2014;2(8):611-620.

50. Su X, Looney M, Robriquet L, Fang X, Matthay MA. Direct visual instillation as a method for efficient delivery of fluid into the distal airspaces of anesthetized mice. Exp Lung Res. 2004;30(6):479-493.

51. Tran CS, et al. The Pseudomonas aeruginosa type III translocon is required for biofilm formation at the epithelial barrier. PLoS Pathog. 2014;10(11):e1004479.

52. Pittet JF, Wiener-Kronish JP, McElroy MC, Folkesson HG, Matthay MA. Stimulation of lung epithelial liquid clearance by endogenous release of catecholamines in septic shock in anesthetized rats. J Clin Invest. 1994;94(2):663-671.

53. Headley MB, et al. Visualization of immediate immune responses to pioneer metastatic cells in the lung. Nature. 2016;531(7595):513-517.

54. Looney MR, Thornton EE, Sen D, Lamm WJ, Glenny RW, Krummel MF. Stabilized imaging of immune surveillance in the mouse lung. Nat Methods. 2011;8(1):91-96

55. Bernard GR, et al. The American-European Consensus Conference on ARDS. Definitions, mechanisms, relevant outcomes, and clinical trial coordination. Am J Respir Crit Care Med. 1994;149(3 Pt 1):818-824.

56. Force ADT, et al. Acute respiratory distress syndrome: the Berlin Definition. JAMA. 2012;307(23):2526-2533. 